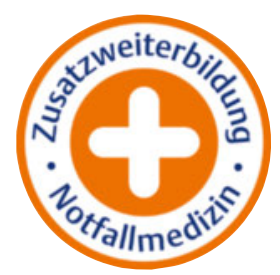

\section{Aktionsplan Sichere Notfallnarkose bei Kindern}

\section{Action Card - Safe RSI (Rapid Sequence Induction)}

Autor Trappe U. ${ }^{1}$, Reifferscheid F. ${ }^{2}$, Thiele J.. Hoedtke J. ${ }^{4}$, Jung P. ${ }^{5}$

Institute ${ }^{1}$ Klinikf. Anästhesie, Rettungs-, Intensivmedizin, Schmerztherapie BG-Unfallkrankenhaus Hamburg

${ }^{2}$ Klinik für Anästhesiologie und Operative Intensivmedizin und Institut für Rettungs- und Notfallmedizin, Universitätsklinikum Schleswig-Holstein, Campus Kiel

${ }^{3}$ Institut für Notfallmedizin, Asklepios Klinikum Harburg, Hamburg

${ }^{4}$ Anästhesiologie und operative Intensivmedizin, Asklepios Klinik Barmbek

${ }^{5}$ Universitätsklinikum Schleswig-Holstein Campus Lübeck, Klinik für Kinder- und Jugendmedizin

Geteilte Erstautorenschaft: U. Trappe und F. Reifferscheid haben zu gleichen Teilen zur Publikation beigetragen.

Schlüsselwörter

- Notfallnarkose

- Patientensicherheit

- Kindernotfall

- Checkliste

- Fehler

- kognitive Hilfsmittel

- Team Ressource Management

Keywords

- Emergency medicine

- anaesthesio

- pediatric

- patient safety

- cognitive aids

- checklist

- team resource management

Bibliografie

DOI http://dx.doi.org/

10.1055/s-0042-117356

Notarzt 2016; 32: 244-253

(c) Georg Thieme Verlag KG

Stuttgart · New York

ISSN 0177-2309

Korrespondenzadresse

Dr. med. U. Trappe

Klinikf. Anästhesie, Inten-

sivmedizin, Notfallmedizin

und Schmerztherapie

BG-Unfallkrankenhaus

Hamburg

Bergedorfer Str. 10

21033 Hamburg

E-Mail: U.Trappe@bgk-

hamburg.de

\section{Zusammenfassung}

Die Einleitung einer Narkose im Rettungsdienst ist aufgrund der ungünstigen Rahmenbedingungen komplex und mit einem erhöhten Fehlerrisiko verbunden. Basierend auf den aktuellen Empfehlungen der Fachgesellschaften wird ein Aktionsplan zur Indikationsstellung, systematischen Vorbereitung von Team und Material sowie zur sicheren Durchführung von Notfallnarkosen vorgestellt. Im Fokus stehen hierbei ein optimiertes Team Ressource Management und die Anwendung bei pädiatrischen Notfallpatienten. Typische Risiken und Fehler werden analysiert und Empfehlungen zu deren Vermeidung gegeben.

\section{Einleitung}

Zwischenfälle ereignen sich in der Medizin regelmäßig. Aufgrund der ungünstigen Rahmenbedingungen ( $\bullet \mathrm{Abb}$ 1) und der hohen Komplexität besteht in Notfallsituationen ein besonders hohes Fehlerrisiko. In allen kritischen Situationen der Medizin kommt dem strukturierten und abgestimmten Handeln innerhalb des Teams eine erhebliche Bedeutung zu. Klare Ablaufplanung, sorgfältige Organisation des Materials und gelebtes Team-Ressource-Management (im folgenden TRM) erleichtern effektives und sicheres Handeln im Notfall und erhöhen die Patientensicherheit [1-3]. Die Einleitung einer Narkose im Rettungsdienst ist ein Musterbeispiel für derartige Situationen, denn sie ist komplex und, abhängig von der Erfahrung des Teams, gefahrengeneigt. Es erfolgt ein Eingriff in gleich mehrere Vitalfunktionen. Bewusstsein und Atmung werden ausgeschaltet, die Kreislauffunktion wird beeinträchtigt und letzten Endes besteht bei jeder Narkose die Gefahr einer maßnahmenimmanenten Verschlechterung des Patientenzustandes.

\section{Summary}

Adverse events are reported to take place regularly during routine medical treatment. Providing safe medical treatment at the site of emergency is an even greater challenge because situations and circumstances are often complex and require multi-team efforts. Induction and maintenance of anaesthesia are an excellent example of complex and dangerous tasks in emergency medicine and have recently been commented on by expert pannels. This article focusses on problems, pitfalls and solutions during induction of emergency anaesthesia and suggests the use of checklists to improve patient safety. Such a checklist is presented step by step focussing on pediatric patients.

Systematische Analysen und Reportingsysteme wie „CIRS-Notfallmedizin“ konnten bestimmte Konstellationen identifizieren, die das Eintreten kritischer Ereignisse im Rahmen von präklinischen Narkosen begünstigen [4]. Die in der Literatur beschriebenen erhöhten Raten ösophagealer Fehlintubationen (bis zu 12\% der Fälle) oder misslungener Intubationsversuche (bis zu 15\%) lassen sich nicht uneingeschränkt auf die aktuelle Situation im deutschen Rettungsdienst übertragen. Dennoch ist auch im notarztbasierten Rettungsdienst mit einer erhöhten Inzidenz schwieriger Atemwegssituationen, unerkannten endobronchialen und ösophagealen Fehllagen zu rechnen, zumal nicht alle Notärzte die gleiche Routine und den gleichen Trainingsstand bzgl. präklinischer Narkosen haben [5, 6]. Handlungsempfehlungen für präklinisches Atemwegsmanagement und die S1Leitlinie Prähospitale Notfallnarkose beim Erwachsenen tragen dem Rechnung [7-10]. Sie fokussieren auf erwachsene Notfallpatienten. Kindernarkosen sind im Rettungsdienst erheblich seltener als Narkosen beim 


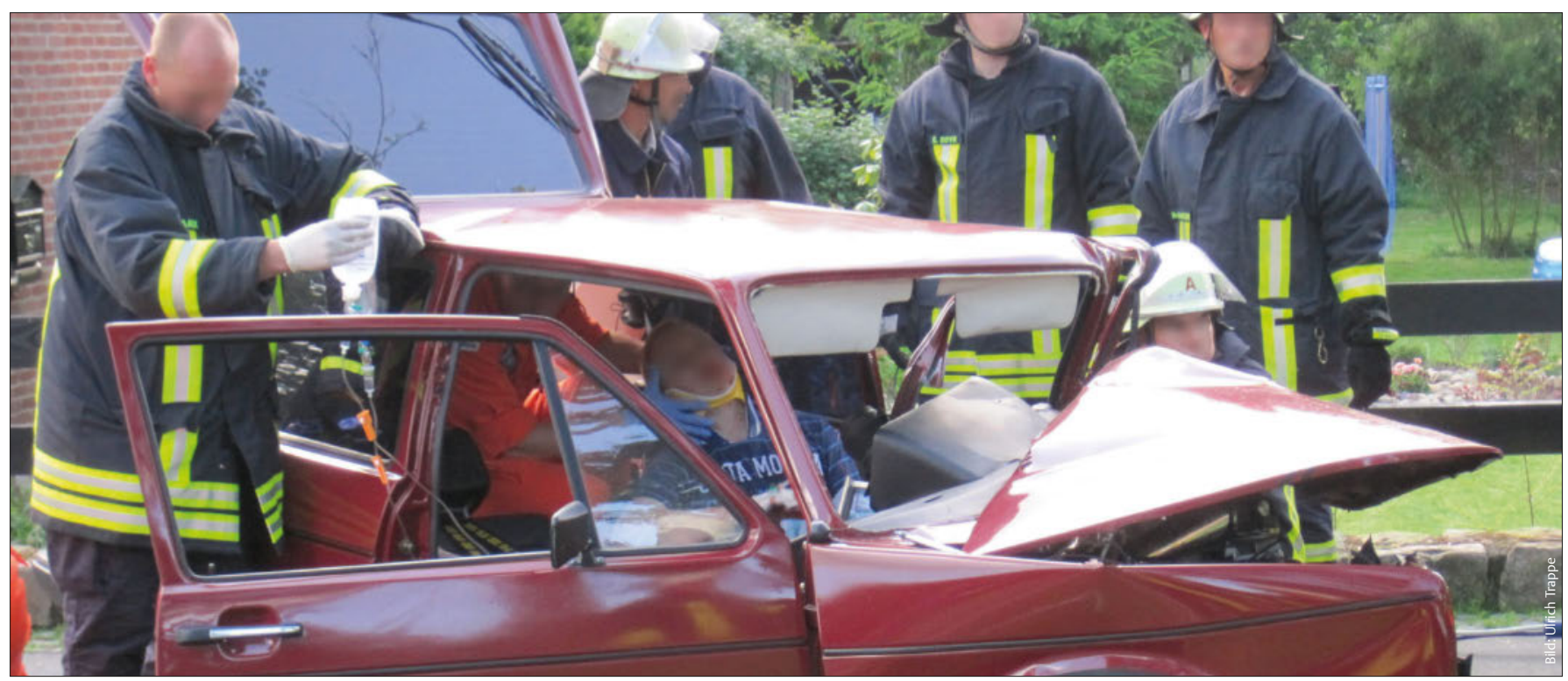

Abb. 1 Narkosearbeitsplatz mit ungünstigen Rahmenbedingungen.

Erwachsenen, wodurch eine ausreichende klinische Routine allein durch die notärztliche Praxis weder erworben noch aufrechterhalten werden kann $[11,12]$. Je jünger das zu behandelnde Kind, desto größer sind Unsicherheit und Stress im Rettungsteam [13]. Folgerichtig wird die Indikation zur Narkoseeinleitung bei Kindern im Rettungsdienst enger gefasst als bei erwachsenen Traumapatienten. Die eigenen Fähigkeiten und Grenzen müssen realistisch eingeschätzt werden, um eine Gefährdung des Patienten zu vermeiden.

\section{Ziele prähospitaler Notfallanästhesie:}

1. Sicherstellung adäquater Oxygenierung, $\mathrm{CO}_{2}$-Elimination und Organperfusion

2. Reduktion des Sauerstoffverbrauchs

3. Organprotektion, insbesondere von Hirn und Herz

4. Sicherung der Atemwege und Schutz vor Aspiration

5. Schmerztherapie, Anxiolyse, psychovegetative Abschirmung

\section{Vorgehen nach dem ABCDE-Schema}

Je jünger Kinder sind, desto eher sind sie im Rahmen einer Anästhesie von Hypoxie und Hypotonie bedroht. Nach dem Leitsatz „Treat first what kills first“" wird, wie in allen maßgeblichen Konzepten zur medizinischen Notfallversorgung (vgl. AHA, ERC etc.), das Vorgehen strukturiert, um lebensbedrohliche Störungen der Vitalfunktionen sofort zu erkennen und priorisiert zu behandeln.

- A: Airway und C-Spine Protection

- B: Breathing

- C: Circulation

D: Disability

- E: Environment/Exposure

Aktionsplan Sichere Notfallnarkose Checklisten finden in HighReliability-Settings wie der Luftfahrt sowohl bei Routineprozeduren als auch in Akut- und Krisensituationen gleichermaßen Anwen- dung. In der Humanmedizin ist beispielhaft die „WHO-Checklist for Patient Safety" erfolgreich etabliert, wenngleich in der Akut- und Notfallmedizin noch weitgehend auf den Gebrauch von Checklisten verzichtet wird. Der hier vorgestellte Aktionsplan Sichere Notfallnarkose (RSI) in Form einer Checkliste soll bei Anwendung laut vorgelesen und jeder Punkt nach durchgeführtem Check abgehakt werden, um maximale Teamperformance zu erreichen und Fehler zu minimieren.

\section{Checkliste}

\section{Präoxygenierung/Positionierung starten}

$\square$ „Kritischer Abfall von Sauersättigung und Kreislauf sind vermeidbar!“

$\square$ Präoxygeniere ab sofort mit $100 \% \mathrm{O}_{2}$ (max. Flow, min. 3 min), Oberkörper erhöht

$\square$ „Alle Teammitglieder handeln vorausschauend und äußern jederzeit Bedenken oder erkannte Gefahren!“

Präoxygenierung Die umgehende Insufflation von High-Flow Sauerstoff über eine dichtsitzende Gesichtsmaske im Rahmen der strukturierten Traumaversorgung ist rettungsdienstliche Routine und steht daher in der Realität meist weit vor der Entscheidung für oder gegen eine Narkoseeinleitung. Der Einsatz von NichtrückatemMasken mit $\mathrm{O}_{2}$-Reservoir erhöht die Effektivität ebenso wie der Gebrauch von Demandventilen. Während bei Kindern ein SauerstoffFlow von 101/min das AMV übersteigt (=High-Flow), ist dazu bei Erwachsenen ein Flow von 151/min notwendig [14]. Die Effektivität der Präoxygenierung lässt sich durch ausreichende Dauer (mindestens $3 \mathrm{~min}$ ) und leichte (beim Traumapatienten achsengerechte) Oberkörperhochlagerung im Sinne einer Anti-Trendelenburg-Lagerung $\left(20-30^{\circ}\right)$ ohne negative Auswirkungen auf den Intubationssitus steigern, wenn die Hämodynamik des Patienten und Fahrzeugtechnik (Trage /-tisch) dies zulassen $[2,15]$. 
Patientensicherheit Dem TRM kommt in dieser kritischen Phase entscheidende Bedeutung zu. Alle Teammitglieder werden aktiv aufgefordert, während des gesamten folgenden Prozesses wachsam und aktiv zur Patientensicherheit beizutragen [16].

Nur gemeinschaftliches vorausschauendes Handeln und die Bereitschaft jedes einzelnen Teammitgliedes jederzeit Bedenken und Gefahren zu kommunizieren („speak up!“) gewährleistet bestmögliche Patientensicherheit.

Das Behandlungskonzept muss an die verfügbaren Ressourcen angepasst sein und umfasst apparative, personelle wie aber auch fachliche Möglichkeiten und Grenzen.

Absolute und relative Indikationen zur Narkoseinduktion Unter den jährlich ca. 1500 schwerverletzten Kindern im Alter bis zu 15 Jahren sind ca. $30 \%$ bis zu 5 Jahre alt. Schwere Kopfverletzungen

\section{Checkliste}

\section{Kritische Überprüfung der Narkoseindikation}

Erfolg ist abhängig von der Expertise der Durchführenden

$\square$ Ist Unterstützung verfügbar?

$\square$ Hinweise auf schwierigen Atemweg oder Muskelerkrankung?

(AIS $\geq 3$ ) machen je nach Altersgruppe 67-78\% aus [17]. Als Goldstandard für die Atemwegssicherung bei erwachsenen Polytraumapatienten gilt die endotracheale Intubation [12]. Bei Kindern mit vermutetem Polytrauma lassen sich absolute von relativen Indikationen für eine Narkoseinduktion am Unfallort unterscheiden ( $\bullet$ Tab. 1). So wird zwischen akuten Störungen der Oxygenierung (A- und
B-Probleme) mit unmittelbarem Handlungszwang und subakuten Gefahren wie Aspirationsgefahr (als Folge von D-Problemen) differenziert. Zumeist lassen sich akute Störungen überbrückend mit einfachen nichtinvasiven Mitteln beseitigen (z.B. Freimachen der Atemwege, Esmarch-Handgriff).

Bessert sich die Oxygenierung trotz Sauerstoffgabe und Freimachen der oberen Atemwege nicht, so liegt kein A-Problem, sondern ein BProblem vor.

Während die insuffiziente Eigenatmung mittels assistierter Beatmung (assistierte Maskenbeatmung/Nicht-invasive-Beatmung) rekompensiert wird, gilt es parallel das B-Problem zu identifizieren und zu behandeln ( $\bullet$ Tab. 3). Bei relativer Indikation kann die Einleitung einer Anästhesie nach Güterabwägung aufgeschoben werden (z.B. bis zum Erreichen des Krankenhauses oder bis zum Eintreffen von erfahrener Unterstützung), um möglichst gute Rahmenbedingungen zu schaffen [18]. In die Entscheidung fließen Informationen der „SAMPLER“-Anamnese ( $\bullet$ Infobox) bezüglich Intubationshindernissen, Medikamentenunverträglichkeiten oder vorliegenden

SAMPLER-Anamnese
S Symptome
A Allergien
M Medikamente
P Patientengeschichte
L letzte Nahrungsaufnahme
E Ereignisse in Verbindung mit aktuellem Notfallgeschehen
R Risikofaktoren

Tab. 1 Indikation zur Narkoseeinleitung bei Notfallpatienten

\section{Absolute Indikation Intubation}

A-Problem (Airway)

Atemwegsverlegung oder Atemwegstrauma

B-Problem (Breathing) [1, 15]

- Hypoxie $\left(\mathrm{S}_{\mathrm{p}} \mathrm{O}_{2}<90 \%{ }^{1}\right)$ trotz Sauerstoffgabe und nach Ausschluss Spannungspneumothorax

- respiratorische Insuffizienz (Atemfrequenz > 29) bei schwerem Thoraxtrauma

- Versagen noninvasiver Therapiemaßnahmen

C-Problem (Circulation) [1, 15]

- traumaassoziierte hämodynamische Instabilität (RR$<90 \mathrm{mmHg}^{2,3}$ )

\section{D-Problem (Disability) [15]}

- schweres SHT (GCS<9)

E-Problem (Exposure)

\section{Relative Indikation Intubation}

A- / B- / C-Problem, welches mit noninvasiven Maßnahmen zu beherr-

schen ist [4]

- Trauma im Bereich der oberen Atemwege

- Verdacht auf ausgeprägte Hyperkapnie bei Hypoventilation
D-Problem (reversibel?) [14, 15]

- Vigilanzminderung: z. B. Intoxikation, Hypoglykämie

- eingeschränkte Schluck- und Abwehrreaktionen (Apoplex etc.)

- schwerer Unfallmechanismus

1 = pulsoxymetrisch gemessen, 2 = Wert für den systolischen Blutdruck, $3=70+(2 \times$ Alter in Jahren) [18] 
Muskelerkrankung ebenso ein wie die Erfahrung des Teams mit der Altersgruppe und Auswahl von Transportziel, -dauer und -modus (bodengebunden oder luftgestützt). Insofern sollte die Indikationsstellung zur invasiven Atemwegssicherung bei Kindern in jedem Fall kritisch überprüft werden [9, 12]. Unstrittig ist, dass die primäre Behandlung polytraumatisierter Kinder in einem kindertraumatologischen Referenzzentrum einen Überlebensvorteil bietet [18, 19].

\section{Checkliste}

\section{Lege einen sicheren Zugang zum Kreislauf (i.v./i.o.)}

$\square$ und prüfe ihn z. B. Bolus 2-5 ml / kg (Vollelektrolyt-Lsg.)

Zugang legen Voraussetzung für eine sichere Narkose ist ein sicherer Zugang zum Kreislauf. Sollte innerhalb von 2 min oder 3 Versuchen kein periphervenöser Zugang zu etablieren sein, besteht im Notfall die Indikation zur Anlage eines intraossären Zuganges. Durch einen Flüssigkeitsbolus von 2-5 ml/ kg KG lässt sich bei herzgesunden Kindern die sichere intravenöse Lage des Zugangs überprüfen und darüberhinaus der narkosebedingte Blutdruckabfall abmildern. Bei C-Problemen sollte primär ein Bolus von $20 \mathrm{ml} / \mathrm{kg}$ Körpergewicht balancierter Vollektrolytlösung (VEL) i.v./i.o. verabreicht und ggf. wiederholt werden

Der viel zitierte zweite großlumige venöse Zugang gelingt bei Kindern regelmäßig nicht, führt stattdessen zu Angst, Schmerz und Schrecken und scheint im Zeitalter des intraossären Zugangs von nachgeordneter Bedeutung für die Sicherheit einer Anästhesie bei Kindern im Notfallsetting zu sein.

Seine Notwendigkeit kann ggf. nach erfolgter Narkoseeinleitung und Atemwegssicherung neu bewertet werden.

\section{Checkliste}

\section{Plane die Narkose, nutze Merkhilfen}

$\square$ Medikamente und deren Dosis geklärt:

$\square$ Analgetikum/ Narkotikum/Muskelrelaxans/Sedativum

$\square$ Kristalloid-Bolus/Notfallmedikamente: Atropin / Akrinor/Adrenalin

$\square$ Größen geklärt: Maske/Spatel / Guedel-/ Endotrachealtubus Layrynxtubus oder -maske (LMA bei Kindern)

$\square$ Zielwerte geklärt: HF, RR, $V_{t}, f=$ Beatmungsfrequenz

Eine strukturierte und sachgerecht durchgeführte Narkoseeinleitung senkt das Risiko für Morbidität und Letalität [20]. Ziel ist die Vermeidung von Hypoxie und Hypotonie. Eine ausreichende Narkosetiefe (tiefe Bewusstlosigkeit, Dämpfung von vegetativen Reflexen und Muskelspannung) schafft die notwendige Voraussetzung für eine erfolgreiche Intubation [21] . Eine unzureichende Anästhesie erschwert sowohl Maskenbeatmung als auch Intubation und begünstigt Komplikationen wie Hypoxie und Aspiration. Im Zweifel sollte den Medikamenten der Vorzug gegeben werden, mit denen

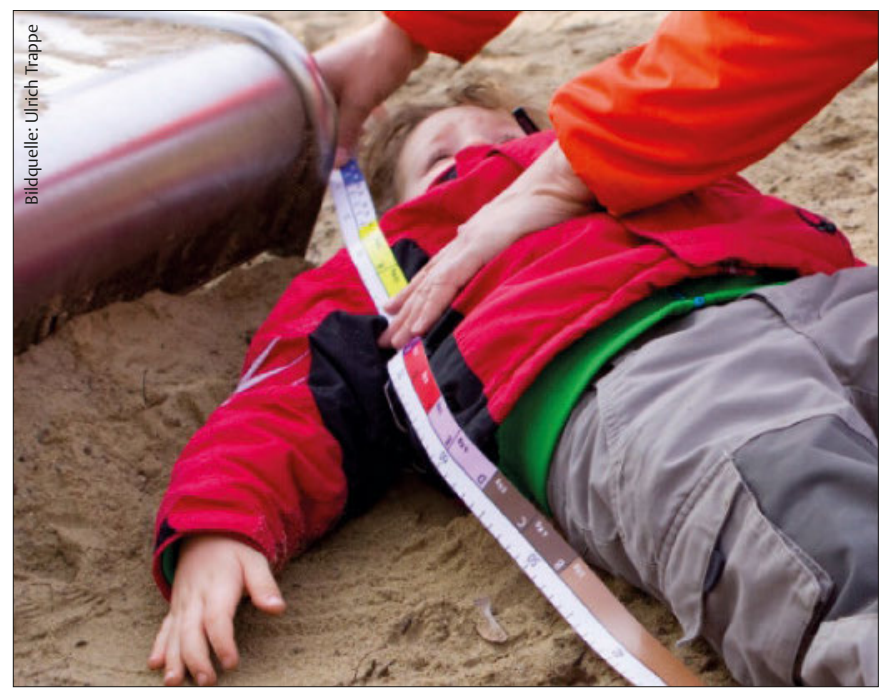

Abb. 2 Anwendungsbeispiel kognitive Hilfsmittel: Abschätzen des Körpergewichtes.

die größte persönliche Erfahrung besteht [22]. Kinder benötigen bezogen auf ihr Körpergewicht im Vergleich zu Erwachsenen deutlich höhere Dosierungen der gängigen Narkosemedikamente. Es empfiehlt sich hier, die Dosierungen anhand des tatsächlichen oder geschätzten Körpergewichtes (Maßbänder, Lineale, etc.) mithilfe sog. kognitiver Hilfsmittel und Datenbanken (z.B. Paulino-System ${ }^{\circledR}$, pädiatrisches Notfalllineal ${ }^{\circledast}$, Gnom ${ }^{\circledR}$, Kindersicher ${ }^{\circledR}$ o.ä.) auszurechnen und festzulegen ( $\bullet$ Abb.2) Ebenso sollte das altersspezifische Medizinequipment (sowie eine Größe kleiner, bzw. größer) ausgewählt und vorbereitet werden.

Muskelrelaxanzien Der Einsatz von Muskelrelaxanzien in der Notfallmedizin wird seit Jahren kontrovers diskutiert. Die Mehrzahl der vorliegenden Untersuchungen zeigt aber, dass die Häufigkeit schwerer Zwischenfälle reduziert werden kann, wenn Relaxanzien zum Einsatz kommen $[4,8,9,23]$. Die Anzahl der Intubationsversuche und deren Dauer lassen sich infolge verbesserter Intubationsbedingungen reduzieren [7, 8, 16, 24]. Die S3-Leitlinie zur Versorgung Schwerverletzter empfiehlt eine Narkoseeinleitung nach den Regeln der „Rapid Sequence Induction“ (RSI) [7]. Auch der Arbeitskreis Kinderanästhesie der DGAI empfiehlt explizit die Einbeziehung von Muskelrelaxanzien in die Notfallnarkose bei Kindern [8, 22]. Selbst bei komatösen Patienten ist zur Intubation eine Anästhesie sinnvoll, da es keine lineare Korrelation zwischen GCS einerseits und Hustenund Würgereflex andererseits gibt.

Auf Grund des raschen Wirkeintritts mit sehr guten Intubationsbedingungen bereits nach ca. 30 s sind Succinylcholin und Rocuronium besonders gut für die Relaxierung von nicht nüchternen Notfallpatienten geeignet.

Unerwünschte Wirkungen Bei allen verwendeten Substanzen muss bei Kindern bis zum Wirkungseintritt zwischenbeatmet werden, da Kinder eine sehr geringe Apnoetoleranz haben. Die muskuläre Erholung dauert zu lange, als dass diese bei Beatmungsproblemen abgewartet werden könnte [6, 20, 23]. 
Während der Anästhesie bei Traumapatienten im Kindesalter ist mit unerwünschten Wirkungen auf die Vitalparameter zu rechnen.

Je kleiner das Kind desto weniger wird das Herz-Zeit-Volumen über die Schlagvolumenvariabilität gesteuert. Bei Neugeborenen und Säuglingen hängt das Herz-Zeit-Volumen fast ausschließlich von der Herzfrequenz ab, während eine Hypovolämie lange Zeit über erhöhten peripheren Gefäßwiderstand und hohe Herzfrequenz kompensiert wird und ganz plötzlich in eine schwere Bradykardie übergeht. Dementsprechend kommt eine Bradykardie von unter 60 Schlägen pro min in dieser Altersgruppe bei Vorliegen von Schockzeichen einem Kreislaufstillstand gleich [14]. In Antizipation dieser typischen, unerwünschten Anästhesiewirkungen bei Traumapatienten wie Bradykardie und/oder Hypotonie, sollten Atropin und Akrinor vorbereitet werden. Um auf den schlimmsten Fall eines Kreislaufstillstandes vorbereitet zu sein, sei auch die Vorbereitung von Suprarenin empfohlen. Die Auswahl der Medikamente zur Narkoseinduktion sollte immer dem Erfahrungsschatz des Anwenders angepasst sein. Bei hämodynamisch instabilen Patienten kann die Einleitung mit Esketamin und Rocuronium weitgehend kreislaufneutral erfolgen [1, 25, 26]. Midazolam wird nach Intubation unter Blutdruckkontrolle zur Sedierung und Prophylaxe ketamininduzierter Halluzinationen verabreicht.

Endotrachealtubus Die Auswahl des Equipments richtet sich nach Alter und Körpergröße des Patienten. Die Anwendung von blockbaren Endotrachealtuben ist bei Kindern aller Altersklassen gefahrlos möglich, insbesondere wenn atraumatisches Material (z.B. Microcuff $^{\odot}$-Tuben) und eine atraumatische Technik (u.a. mit Cuffdruckmessung) verwendet werden [23]. Bis zu einem Körpergewicht von $10 \mathrm{~kg}$ ist die Anwendung ungeblockter Endotrachealtuben durchaus üblich. Keinesfalls darf die endotracheale Intubation erzwungen werden [23].

Larynxmaske- und tubus Während für Erwachsene und Jugendliche Larynxmasken und Larynxtuben gleichwertig als alternative Atemwege (Plan B) zur Verfügung stehen, wird in aktuellen Veröffentlichungen für Kinder aller Altersgruppen die Larynxmaske mit Drainagekanal aufgrund ihrer breiten und gefahrlosen Anwendung in der Kinderanästhesie als alternativer Atemweg empfohlen [27].

Rachentubus In der Pädiatrie ist der nasal eingeführte „Rachentubus“ als supraglottische Beatmungsalternative etabliert und kann bei problematischer Beutel-Masken-Ventilation eine wenig invasive, einfach durchzuführende und suffiziente Alternative darstellen. Hierbei wird ein kleiner ungecuffter Tubus analog zum Wendeltubus durch ein Nasenloch bis in der Hypopharynx vorgeschoben (Distanz: Nasenloch-Kieferwinkel). Unter Zuhalten von Mund und Nase lässt sich eine suffiziente Beutelbeatmung durchführen. Die Anwendung ist jedoch auf Patienten limitiert, bei denen ein Schädel-Hirn-Trauma sicher ausgeschlossen werden kann, da ansonsten eine intrazerebrale Fehlplatzierung zu befürchten ist

\section{Checkliste}

\section{Vermeide Medikationsfehler}

4-Augen-Prinzip angewendet

Spritzen gekennzeichnet

Vorbereitung, Kennzeichnung und richtige Applikation der Medikamente Die Gefahr von Medikationsfehlern in unübersichtlichen Notfallsituationen ist immens. Dokumentierte Fehldosierung von 808\% der errechneten Adrenalindosis vor allem bei Säuglingen und Kleinkindern zwingen uns, im Einsatzalltag alle zur Verfügung stehenden Instrumente zur Verbesserung der Sicherheit zu nutzen [28].

Die gute Vorbereitung und Kennzeichnung sowie die richtige Art der Applikation sind hierfür von entscheidender Bedeutung. Vorbereitung und Kennzeichnung von Medikamenten für die Kinderanästhesie werden erheblich sicherer, wenn die Schritte Medikamentenauswahl, -verdünnung und -kennzeichnung durch eine zweite Person kontrolliert werden (4-Augen-Prinzip).

Die Zuhilfenahme einer Verdünnungsanleitung (sog. kognitive Hilfen wie z.B. Paulino ${ }^{\circledR}$-System, päd. Notfalllineal ${ }^{\circledR}, \mathrm{Gnom}^{\circledR}$, Kindersicher $^{\circledR}$ o.ä.) sowie die standardisierte und den aktuellen Empfehlungen folgende Kennzeichnung der Spritzen (z.B. RETTiketten ${ }^{\odot}$ ) sollten unbedingt genutzt werden ( $\bullet$ Abb. 3). Die Applikation der korrekten Medikamentendosis birgt eine weitere Fehlermöglichkeit und muss durch gute und unmissverständliche Team-Kommunikation abgesichert werden. Zu diesem Zweck sollten immer das Volumen in $\mathrm{ml}$ und die Wirkstoffmenge in mg oder $\mu \mathrm{g}$ angesagt und im Sinne eines

\section{Checkliste}

\section{Mache einen Eqipmentcheck}

$\square$ Absaugung komplett, funktioniert und läuft/Sauerstoff läuft

EKG/SpO mit Pulston/ Kapnografie/RR (Intervall: 3 min)

Guedel-/ Endotrachealtubus (mit Führungsstab) + kleinere Größe

Larynmaske oder -tubus (LMA bei Kindern)

$\square$ Kopf liegt in Neutralposition (ggf. Schulterrolle bei Säugling/ Kleinkind)

jedes Teammitglied kennt seine Aufgabe

manuelle In-Line-Stabilisierung der HWS anstelle HWS-Orthese für die Dauer der Narkoseeinleitung beim Traumapatienten (SHT/HWS-Trauma)

Präoxygenierung „Der polytraumatisierte Patient soll vor Narkoseeinleitung präoxygeniert werden“ (GoR A) [12]. Einschränkend ist hierzu zu sagen, dass bei Kindern die Präoxygenierung mit dichtsitzender Maske nicht erzwungen werden sollte (z.B. wenn sie Angst haben), da Stress die Sauerstoffbilanz unnötig negativ beeinflusst. Stattdessen hat sich ein altersgerechter Zugang zum Patienten durch bewusste Aufmerksamkeitslenkung (z.B. „Tauchermaske“, „Pilotenmaske") in vielen Fällen als hilfreich erwiesen. Je jünger der Patient, desto geringer ist der Effekt einer Präoxygenierung, da das Residu- 


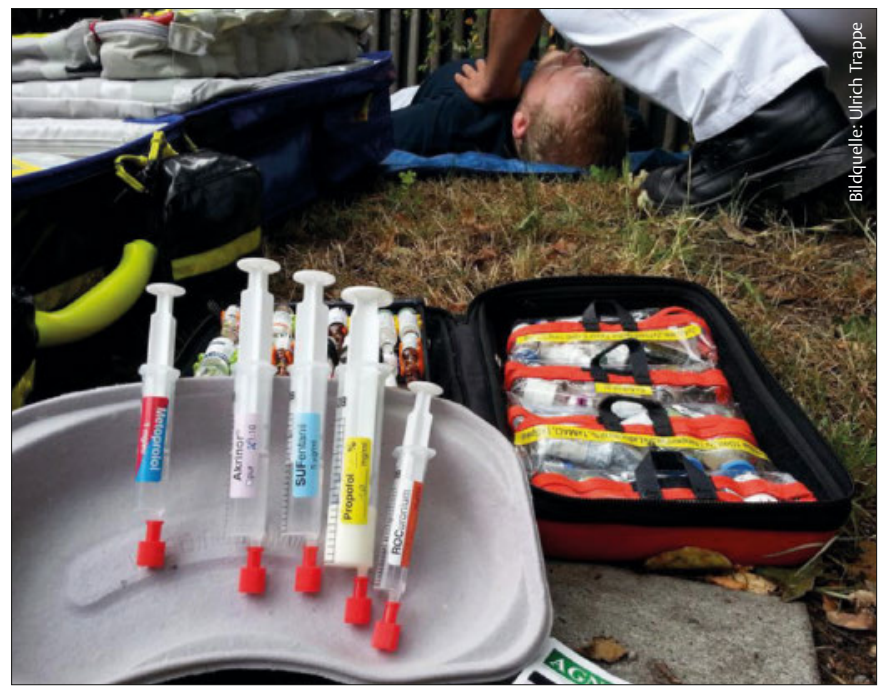

Abb. 3 RETTiketten ${ }^{\circledast}$ : Standardetiketten erhöhen die Patientensicherheit

alvolumen (als „Sauerstoffspeicher“) relativ gesehen kleiner und der Sauerstoffverbrauch relativ gesehen größer ist. Während der Präoxygenierung sind die Funktionalität des Equipments sicherzustellen, das Monitoring zu komplettieren, die Aufgaben im Team zu verteilen und das weitere Vorgehen im Sinne eines Briefings abzustimmen, das auch das Vorgehen bei schwierigem Atemweg einbezieht [9, 12]. Dem TRM kommt hierbei überragende Bedeutung zu. Weiterhin wird der Plan B für realistische kritische Situationen gebrieft.

In-Line-Stabilisierung statt HWS-Orthese Die korrekt angelegte HWS-Orthese erschwert die orale Intubation erheblich (GoR B) [12]. Daher soll sie für die Dauer der Anästhesieinduktion durch eine manuelle In-Line-Stabilisierung der HWS ersetzt werden (GoR B) [15]. Ein Helfer führt die manuelle In-Line-Stabilisierung der HWS durch, so dass ein zweiter die HWS-Orthese gefahrlos öffnen kann. Dies verschafft dem Intubierenden für das Einführen des Laryngoskopspatels in den Rachen und die Laryngoskopie eine größere Mundöffnung bei stabilisierter HWS. Nach Lagekontrolle und Tubusfixierung wird die HWS-Orthese wieder geschlossen und die manuelle In-Line-Stabilisierung beendet.

\section{Checkliste}

\section{Intubation - nach Möglichkeit unter Sicht!}

$\square$ Videolaryngoskop/alternatives Equipment verfügbar und vorbereitet?

Optische Intubationshilfen Grundsätzlich ist im Notarztdienst mit einer erhöhten Prävalenz des schwierigen Atemweges zu rechnen. Daher sind Videolaryngoskopie und andere optische Intubationshilfen in geübter Hand eine wertvolle Ergänzung der Hilfsmittel zur Sicherung der Atemwege und sollten vor Narkoseinduktion vorbereitet werden. Je nach Gerät ist mehr oder weniger Übung erforderlich. Limitiert sind die Einsatzmöglichkeiten insbesondere bei Blutung im Mund-Rachenraum oder Sekretverlegung der oberen Luftwege.
Leider fehlt auf Rettungsmitteln regelmäßig ein Spatel in Kindergröße, sodass beim vermeintlich schwierigen Atemweg im Kindesalter auf das effektivste Hilfsmittel verzichtet werden muss.

\section{Checkliste}

\section{Medikamente: adäquate Dosierung}

$\square$ ausreichende Narkosetiefe mit zusätzlicher Muskelrelaxierung schaffen bessere Intubationsbedingungen (vgl. Punkt 3)

$\square$ appliziere Medikamente entsprechend der Planung und Vorbereitung (s. Punkt 3)

$\square$ achte auf gute und effektive Kommunikation

Die geplanten und vorbereiteten Dosen des Analgetikums, des Hypnotikums und des Muskelrelaxans werden zügig nacheinander injiziert (modifizierte Rapid-Sequenz-Induktion) [22].

Das Auslösen pharyngealer Reflexe (bei einer zu „flachen“ Narkose) durch Nichtabwarten des Wirkungseintrittes der Narkosemedikation ist häufig Ursache für Laryngospasmus oder Erbrechen während einer Narkoseeinleitung und kann vermieden werden [8, 22].

\section{Checkliste}

\section{Bei Kindern immer: sanfte Maskenbeatmung bei RSI ist sicher und vermeidet Hypoxie!}

ca. 20-30 s bie zur vollen Medikamentenwirkung

Insbesondere der Kommunikation und dem TRM kommen herausragende Bedeutung zu, um Medikamente- und/oder Timing-Fehler zu vermeiden ( $\odot$ Abb. 4). „Bei polytraumatisierten (erwachsenen) Patienten soll zur endotrachealen Intubation eine Notfallnarkose aufgrund der meist fehlenden Nüchternheit und des Aspirationsrisikos als Rapid Sequence Induction (RSI) durchgeführt werden“ [12]. Kleine Kinder sind jedoch viel stärker durch eine Hypoxie als durch eine Aspiration gefährdet [22|. Daher hat „die Oxygenierung des Kindes zu jedem Zeitpunkt der Narkose oberste Priorität - das gilt sogar für den Fall der Aspiration!“ [22]. Nicht die rasche endotracheale Intubation steht im Vordergrund, sondern die sichere Narkoseeinleitung des nicht nüchternen Kindes. Hierzu gehören die rasche Induktion einer tiefen Anästhesie, eine optimale Oxygenierung bis zur suffizienten Muskelrelaxierung und schließlich die atraumatische Atemwegssicherung (ohne jegliche Gegenwehr) [22].

„Die klassische RSI ohne Zwischenbeatmung führt bei Neugeborenen, Säuglingen und Kleinkindern meist unvermeidbar zur Hypoxämie. Um diese gefährliche Situation mit dem Risiko von weiteren Sekundärschäden zu vermeiden, muss ein Kind daher in der sensiblen Phase zwischen Narkoseinduktion und vollständiger Muskelrelaxierung vorsichtig mit Beutel und Maske beatmet werden“, sodass von einer modifizierten RSI gesprochen wird [22]. 
Die Maskenbeatmung erfolgt mit maximaler Sauerstoffkonzentration nur so stark, dass sich der Thorax gerade eben sichtbar hebt. Durchgeführt wird sie ohne hohen Druck (max. 10-12 $\mathrm{cm} \mathrm{H}_{2} \mathrm{O}$ ), damit eine Überblähung bzw. Luftinsufflation in den Magen vermieden wird [22|. Erst nach ca. 20-30s ist mit der vollen Medikamentenwirkung und den damit einhergehenden bestmöglichen Intubationsbedingungen zu rechnen. Die Lagerung des Kopfes entscheidet über den Erfolg der Beatmungs- und Intubationsbemühungen. Bei Kindern sollte der Kopf in der Neutralposition fixiert werden. Im Säuglingsalter ist hierfür wegen des relativ großen Hinterkopfes regelhaft ein Unterpolstern der Schultern notwendig. Gelingt die endotracheale Intubation nicht im ersten Versuch, erfolgt die sofortige intermittierende Maskenbeatmung und optimierende Vorbereitung weiterer Intubationsversuche. Der Krikoiddruck zur Vermeidung von Aspiration kann heute nicht mehr empfohlen werden [22|.

Grundregeln zur Vermeidung eines Intubationstraumas

1. Die Benutzung eines Führungsstabes erleichtert die endotracheale Intubation. Der Führungsstab überragt die Tubusspitze nicht.

2. Der Tubus wird ohne Widerstand platziert, anderenfalls wird auf die nächstkleinere Tubusgröße ausgewichen.

3. Nach Intubation wird die Tubuslage unter Sicht durch Zurückziehen korrigiert, um eine zu tiefe und einseitige Lage auszuschließen.Cave: insbesondere bei kleinen Kindern kann aus anatomischen Gründen ein zu tiefer und damit einseitig einliegender Tubus sowohl rechts-, als auch linksseitig zu liegen kommen.

4. Anschließend wird der Cuff geblockt, der Cuffdruck muss kontrolliert werden.

\section{Checkliste}

\section{A- / B-Probleme? Vorwärtsstrategie}

$\square$ sanfte Zwischenbeatmung mit Beutel/Maske $<12 \mathrm{~cm} \mathrm{H}_{2} \mathrm{O}$

$\square$ A-Problem: variiere Kopfposition/Esmarch-Griff/ doppelter

C-Griff/Larynxmaske bei Kindern, ggf. Larynxtubus bei Erwachsenen

Insbesondere pädiatrische Traumapatienten sterben nicht daran, dass sie nicht intubiert werden können, sondern daran, dass sie nicht beatmet werden.

Während der anatomisch bedingte schwierige Atemweg bei pädiatrischen Traumapatienten eine Rarität ist, stellt in dieser Patientengruppe die funktionelle Atemwegsverlegung ein zentrales Problem dar [23]. Ist der geplante Atemweg nicht zu realisieren, wird umgehend ein supraglottischer Atemweg etabliert. Die endotracheale Intubation ist (bei Kindern) nicht in jedem Fall der Goldstandard und darf nicht erzwungen werden [zitiert nach 23]. Wenn vorhanden, kann eine alternative Technik genutzt werden (vgl. Punkt 7). Sollte eine Oxygenierung mit nichtinvasiven Maßnahmen nicht zu gewährleisten sein, steht bei Erwachsenen mit der Koniotomie eine invasive Option zur Verfügung.

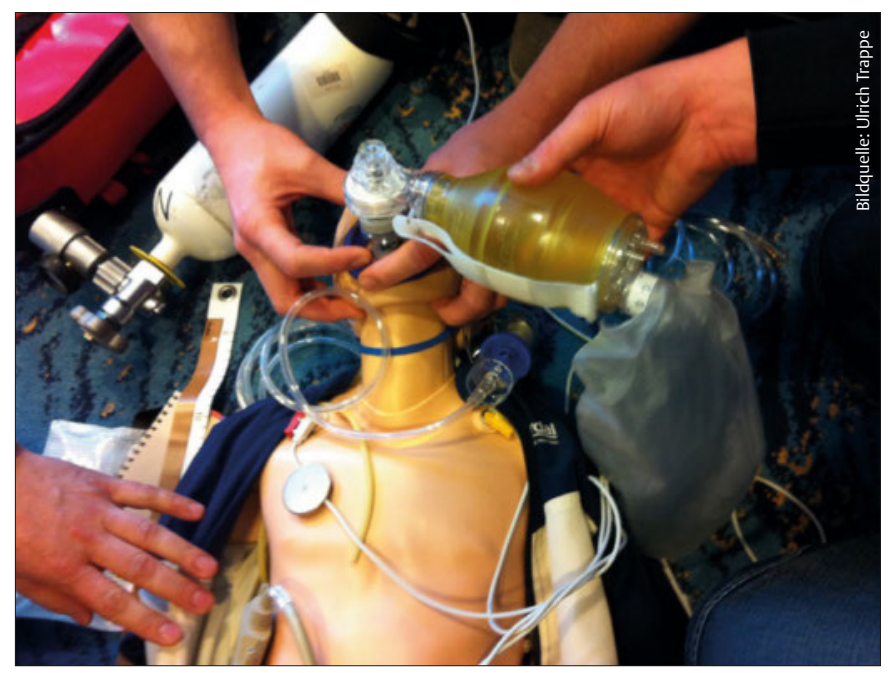

Abb. 4 Narkoseeinleitung ist Teamarbeit und Teamarbeit muss trainiert werden!

Bei kleinen Kindern stellt die Koniotomie oder auch die Tracheotomie weniger eine realistische Handlungsoption, als vielmehr eine Verzweiflungstat dar.

\section{Checkliste}

11. Bei Problemen: Die systematische Fehlersuche im Rahmen einer Reevaluation wird durch Akronyme erleichtert. Reevaluation nach D O PES und A B CDE

Ist die Oxygenierung trotz erfolgter Intubation und Beatmung gestört, muss eine systematische Fehlersuche erfolgen, um das Problem zu lösen. Eine Sauerstoffsättigung über 94 \% ist anzustreben! Durch eine periphere O2-Sättigung von mindestens 90\% wird zumindest eine schwere Hypoxie vermeiden. Die Ursachensuche richtet sich nach dem ABCDE-Schema. Eine häufige Ursache schlechter peripherer Saustoffsättigung ist ein mögliches C-Problem im Sinne eines niedrigen Blutdrucks, welches sich anhand der Plethysmographie-Kurve identifizieren lässt.

Gemäß dem Akronym DOPES lassen sich Probleme bei beatmeten Patienten systematisch und effektiv lösen ( $\bullet$ Tab. 2).

\section{Checkliste}

12. Erhalte die Narkose aufrecht, beatme protektiv

AZV: $6-8 \mathrm{ml} / \mathrm{kg}$, Pinsp. und $\mathrm{FiO}_{2}$ titrieren, Ziel-SpO 2 : mind. 94\%

$\square$ et $\mathrm{CO}_{2}: 35 \mathrm{mmHg}$, Respiratorbeatmung* erst $>10 \mathrm{~kg} \mathrm{KG}$ sicher möglich

kontinuierliche Aufrechterhaltung der Narkose mittels Perfusor

( ${ }^{*}$ aktuelle Notfallrespiratoren im Rettungsdienst) 
Tab. 2 Ursachen für A- und B-Probleme bei beatmeten Patienten: DOPES-Akronym

\begin{tabular}{|c|c|c|c|c|}
\hline & Begriffserklärung & Definition des Problems & Differenzialdiagnose & Maßnahmen \\
\hline D & Dislokation & $\begin{array}{l}\text { Akzidentelle Lageänderung } \\
\text { einer Atemwegshilfe (Tubus, } \\
\text { Larynxmaske) }\end{array}$ & $\begin{array}{l}\text { Dislokation von Tubus oder } \\
\text { Larynxmaske } \\
\text { - einseitige Ventilation }\end{array}$ & $\begin{array}{l}\text { Aaßßere Inspektion der Lage der } \\
\text { Atemwegshilfe } \\
\text { - Auskultation } \\
\text { - laryngoskopische Kontrolle der } \\
\text { Tubuslage } \\
\text { - Lagekorrektur }\end{array}$ \\
\hline $\mathbf{P}$ & Pulmo & $\begin{array}{l}\text { Beeinträchtigung der Ventila- } \\
\text { tion und/ oder des Gasaus- } \\
\text { tauschs }\end{array}$ & $\begin{array}{l}\text { - Gegenatmen, Pressen, Hus- } \\
\text { ten } \\
\text { - Bronchospasmus } \\
\text { - Atelektasen } \\
\text { - Lungenkontusion } \\
\text { - Pneumonische Infiltrate } \\
\text { - Aspiration } \\
\text { - Lungenödem } \\
\text { - Pleuraerguss } \\
\text { - Pneumothorax } \\
\text { - Hämatothorax }\end{array}$ & $\begin{array}{l}\text { - Überprüfung und Anpassung der } \\
\text { Beatmungsparameter und der Anästhesietiefe } \\
\text { Spezifische Therapien: } \\
\text { - Bronchospasmolyse } \\
\text { - Rekrutierung der Lunge } \\
\text { - ggf. permissive Hyperkapnie } \\
\text { Diuresesteigerung } \\
\text { - Behandlung kardialer Ursache } \\
\text { - Thoraxdrainage } \\
\text { - (Antibiotikagabe) } \\
\text { (Bronchoskopie }\end{array}$ \\
\hline$E$ & Equipment & Gerätefehler & $\begin{array}{l}\text { - mechanische Fehler } \\
\text { - Kalibrierungsfehler } \\
\text { - Fehler in der Elektronik }\end{array}$ & $\begin{array}{l}\text { Diskonnektion des Patienten vom Beatmungs- } \\
\text { gerät und } \\
\text { - Beatmung mit Beatmungsbeutel } \\
\text { - Überprüfung, Korrektur oder Austausch des } \\
\text { Beatmungsgeräts }\end{array}$ \\
\hline $\mathrm{S}$ & Stomach & $\begin{array}{l}\text { Verminderung der Compli- } \\
\text { ance der Lunge auf Grund } \\
\text { von erhöhtem intraabdomi- } \\
\text { nellem Druck } \\
\text { Intraplumonaler Rechts-Links- } \\
\text { Shunt }\end{array}$ & $\begin{array}{l}\text { Distension des Magens } \\
\text { oder Darms durch Luft, } \\
\text { Flüssigkeit, Nahrung oder } \\
\text { Stuhl } \\
\text { - Freie Flüssigkeit oder Luft } \\
\text { im Abdomen Enterothorax } \\
\text { - Kapnoperitoneum } \\
\text { - pulmonal-vaskuläre } \\
\text { Ursachen } \\
\text { - erhöhter PAP }\end{array}$ & $\begin{array}{l}\text { Deatmungsdrücke } \\
\text { - anpassen, PEEP erhöhen } \\
\text { - Magensonde legen und } \\
\text { - absaugen } \\
\text { - Narkosetiefe und } \\
\text { - Relaxierungsgrad } \\
\text { - optimieren } \\
\text { - differenzierte Katecholamintherapie }\end{array}$ \\
\hline
\end{tabular}


Auch in der Notfallmedizin ist eine lungenprotektive Beatmung mit physiologischen Tidalvolumina anzustreben. Das Atemminutenvolumen sollte anhand des etCO $\mathrm{CO}_{2}$ gesteuert werden. Ziel ist die Normoventilation mit Normoxie $\left(\mathrm{SpO}_{2}: \geq 94 \%\right)$ und Normokapnie $\left(\right.$ etCO $\mathrm{C}_{2}$ : $35 \mathrm{mmHg}$ ) (GoR A) [12]. Allerdings kann bei Traumapatienten zwischen dem endexpiratorisch gemessenen et $\mathrm{CO}_{2}$ und dem pulmonalarteriellen $\mathrm{pCO}_{2}$ aufgrund von Ventilations-, Diffusions- oder Perfusionsstörungen eine erhebliche Differenz bestehen. Daher sollte das etCO ${ }_{2}$ stets in Verbindung mit dem AMV und den Kreislaufverhältnissen interpretiert werden [12]. Die Beatmung von Kindern mit einem Körpergewicht $\geq 10 \mathrm{~kg}$ ist mit den meisten marktüblichen Beatmungsgeräten für den Rettungsdienst zuverlässig möglich (minimales Vt $50 \mathrm{ml}$ ). Unterhalb dieser Grenze ist eine Handbeatmung mit kapnographischer Kontrolle zu empfehlen.

Insbesondere bei längeren Transportzeiten und dem Einsatz länger wirksamer Muskelrelaxanzien ist eine kontinuierliche Aufrechterhaltung der Narkose mittels Perfusor notwendig. Im notfallmedizinischen Setting können Zeichen unerwünschter Wachheit insbesondere nach Muskelrelaxierung unentdeckt bleiben. Die Wirkdauer einer Einzelbolusgabe von Narkosemitteln variiert (und wird im Stress möglicherweise nicht rechtzeitig wiederholt) [18, 25].

\section{Checkliste}

\section{Temperaturkontrolle}

Vermeide akzidentelle Hyothermie

Wärmemanagment Patienten in Narkose sind regelmäßig von einer mehr oder minder schweren Hypothermie bedroht. Insbesondere Kinder kühlen in Narkose schnell aus (große Körperoberfläche/Kopf=Wärmeverlust im Vergleich zur Wärmeproduktion=Körpervolumen). Sämtliche physiologischen Mechanismen zur Autothermoregulation sind unter Allgemeinanästhesie vermindert. Bei allen narkotisierten Notfallpatienten ist auf ausreichenden Wärmeerhalt zu achten. Eingeklemmte Unfallopfer sind während verlängerter Rettungszeiten einem besonders großen Risiko ausgesetzt. Diesem Umstand trägt auch das E für Exposure Rechnung. Bei Traumapatienten kommt dem Wärmemanagement eine besonders große Bedeutung zu, denn durch Kälte wird der Sauerstoffverbrauch erhöht und es können Blutgerinnungsstörungen, erhöhte Infektionsanfälligkeit und unerwünschte kardiale Ereignisse auftreten. Nur durch aktives Wärmemanagement kann eine akzidentelle, eventuell schwere Hypothermie vermieden werden.

\section{Kernaussagen}

- Die Narkoseeinleitung und Atemwegssicherung ist bei allen Notfallpatienten - insbesondere bei Kindern mit einem erhöhten Risiko verbunden.

- Die Indikation zur Einleitung einer Narkose bei Kindern im präklinischen Setting sollte kritisch gestellt werden.

- Nichtinvasiven Maßnahmen zur Stabilisierung der Vitalfunktionen gebührt im Zweifel der Vorzug.

- Bei sorgfältiger Indikationsstellung und Durchführung trägt die Narkose bei kindlichem Polytrauma zur Prognoseverbesserung bei.

- Erfahrung, insbesondere in der Kernkompetenz Atemwegsma nagement und regelmäßiges Teamtraining sind der Schlüssel zur erfolgreichen Kindernarkose im Notfall.

- Um die Patientensicherheit zu erhöhen können kognitive Hilfen, gelebtes und effektiv angewendetes TRM einen wichtigen Beitrag leisten.

- Die vorgeschlagene Checkliste für die Narkoseeinleitung ist ein Hilfsmittel, an dem sich das TRM orientieren kann.

Interessenkonflikt

U. Trappe: Urheber Paulino-System für die Kindernotfallmedizin. alle anderen: keine

Literatur

1 Sibley A, Mackenzie M, Bawden J et al. A prospective review of the use of ketamine to facilitate endotracheal intubation in the helicopter emergency medical services (HEMS) setting. Emerg Med J 2011; 28: 521-525

2 Martinon C, Duracher C, Blanot S et al. Emergency tracheal intubation of severely head-injured children: changing daily practice after implementation of national guidelines. Pediatr Crit Care Med 2011;12: 65-70

3 Hofmeister E, Quandt J, Braun C et. al. Development, implementation and impact of simple patient safety interventions in a university teaching hospital. Veterinary Anaesthesia and Analgesia 2014; 41: 243-248

4 Hohenstein C, Schultheis K, Winning K et al. Kritische Zwischenfälle im Atemwegsmanagement der präklinischen Notfallmedizin. Anästhesist 2013; 63: 720-727

5 Reifferscheid F, Aschenbrenner $U$ et al. Airway management in out of hospital cardiac arrest. Resuscitation 2014; 85: S33

6 Timmermann A, Eich C, Russo S. Prehospital airway management: a cprospective evaluation of anaesthesia trained emergency physicians. Resuscitation 2006; 70; 179-185

7 Bernhard M, Matthes G, Kanz KG et al. Notfallnarkose, Atemwegsmanagement und Beatmung beim Polytrauma - Hintergrund und Kernaussagen der S3-Leitlinie Polytrauma. Anästhesist 2011; 60: 1027-1040

8 Russo SG, Trieschmann U, Nicolai T. Atemwegsmanagement bei Kindern in Notfallsituationen. Notfall Rettungsmed 2014; 17: 105-112

9 Timmermann A, Byhahn C, Wenzel V et al. Handlungsempfehlungen für das präklinische Atemwegsmanagement. Anästh Intensivmed 2012; 53: 294-308

10 AWMF-Register 001-030 (2015) S1-Leitlinie Prähospitale Notfallnarkose beim Erwachsenen

11 Helm M, Bien G, Lampl L. Pädiatrischer Notfallpatient im Luftrettungsdienst Einsatzrealität unter besonderer Berücksichtigung invasiver Maßnahmen. Anästhesist 2010; 10: 896-903

12 S3-Leitlinie Polytrauma/Schwerverletzten-Behandlung der DGU 2011. AWMF online Registernr. 012-019 (Gültigkeit verlängert bis 30.06.2016).

13 Eich C, Roessler M, Timmermann A et al. Präklinische Kindernotfälle - Notärztliche Wahrnehmung und Einschätzung. Anästhesist 2009; 58: 876-883

14 American Heart Association, Pediatric Advanced Life Support, Provider Manual, AHA 2011 


\section{Aktionsplan Sichere Notfallnarkose}

\section{Präoxygenierung/Positionierung starten}

$\square$ „Kritischer Abfall von Sauersättigung und Kreislauf sind vermeidbar!“

$\square$ Präoxygeniere ab sofort mit $100 \% \mathrm{O}_{2}$ (max. Flow, min. 3 min), Oberkörper erhöht

$\square$ „Alle Teammitglieder handeln vorausschauend und äußern jederzeit Bedenken oder erkannte Gefahren!“

\section{Kritische Überprüfung der Narkoseindikation}

Erfolg ist abhängig von der Expertise der Durchführenden

$\square$ Ist Unterstützung verfügbar?

$\square$ Hinweise auf schwierigen Atemweg oder Muskelerkrankung?

\section{Lege einen sicheren Zugang zum Kreislauf (i.v./i.o.)}

$\square$ und prüfe ihn z. B. Bolus 2-5 ml / kg (Vollelektrolyt-Lsg.)

\section{Plane die Narkose, nutze Merkhilfen}

$\square$ Medikamente und deren Dosis geklärt:

$\square$ Analgetikum/Narkotikum/Muskelrelaxans/Sedativum

$\square$ Kristalloid-Bolus/Notfallmedikamente: Atropin / Akrinor/Adrenalin

$\square$ Größen geklärt: Maske/Spatel/ Guedel-/ Endotrachealtubus Layrynxtubus oder -maske (LMA bei Kindern)

$\square$ Zielwerte geklärt: HF, RR, $V_{\mathrm{t}}, \mathrm{f}=$ Beatmungsfrequenz

\section{Vermeide Medikationsfehler}

$\square$ 4-Augen-Prinzip angewendet

$\square$ Spritzen gekennzeichnet

\section{Mache einen Eqipmentcheck}

$\square$ Absaugung komplett, funktioniert und läuft/Sauerstoff läuft

EKG $/ \mathrm{SpO}_{2}$ mit Pulston / Kapnografie / RR (Intervall: 3 min)

Guedel-/ Endotrachealtubus (mit Führungsstab) + kleinere Größe

Larynmaske oder -tubus (LMA bei Kindern)

$\square$ Kopf liegt in Neutralposition (ggf. Schulterrolle bei Säugling/

Kleinkind)

$\square$ jedes Teammitglied kennt seine Aufgabe

$\square$ manuelle In-Line-Stabilisierung der HWS anstelle HWS-Orthese für die Dauer der Narkoseeinleitung beim Traumapatienten

(SHT/HWS-Trauma)

15 Klandelwal N, Khorsand S, Mitchell S et al. Head elevated Patient positioning decreases complications of emergent Tracheal intubation in the ward and intensive care unit. Anaesth Analg 2016; 4: 1101-1107

16 S2k-Leitlininie Schädel-Hirn-Trauma, AWMF-Register 024/018

17 Deutsche Gesellschaft für Unfallchirurgie (Hrsg). Weißbuch Schwerverletzen- Versorgung. Stuttgart: Thieme-Verlag 2012

18 Schöneberg C, Schweiger B, Metzelder M et al. Das verletzte Kind - Diagnostisches Vorgehen im Schockraum. Notfall Rettungsmed 2014; 17: 255-268

19 Jakob H, Wyen H, Marzi l et al. Polytrauma im Kindesalter - Management, Diagnostik- und Therapiestrategien; Trauma Berufskankh 2013; 15: S67-S74

20 Paal P, Herff H, Mitterlechner et al. Anaesthesia in prehospital emergencies and in the emergency room. Resuscitation 2010; 81: 148-164

21 Smith KA Gothard MD, Schwartz HP et al. Risk Factors for Failed Tracheal Intubation in Pediatric and Neonatal Critical Care Speciality Transport. Prehosp Emerg Care 2015; 19: 17-22

22 Schmidt J, Strauß JM, Becke K et al. Handlungsempfehlung zur Rapid-SequenceInduction im Kindesalter. Anaesthlntensivmed 2007; 48: 88-93

\section{Intubation - nach Möglichkeit unter Sicht!}

$\square$ Videolaryngoskop / alternatives Equipment verfügbar und vorbereitet?

\section{Medikamente: adäquate Dosierung}

$\square$ ausreichende Narkosetiefe mit zusätzlicher Muskelrelaxierung schaffen bessere Intubationsbedingungen (vgl. Punkt 3)

$\square$ appliziere Medikamente entsprechend der Planung und Vorbereitung (s. Punkt 3)

$\square$ achte auf gute und effektive Kommunikation

\section{Bei Kindern immer: sanfte Maskenbeatmung bei RSI ist sicher und vermeidet Hypoxie!}

ca. 20-30 s bie zur vollen Medikamentenwirkung

\section{A-/ B-Probleme? Vorwärtsstrategie}

$\square$ sanfte Zwischenbeatmung mit Beutel/Maske $<12 \mathrm{~cm} \mathrm{H}_{2} \mathrm{O}$

A-Problem: variiere Kopfposition/Esmarch-Griff/ doppelter C-Griff/Larynxmaske bei Kindern, ggf. Larynxtubus bei Erwachsenen

11. Bei Problemen: Die systematische Fehlersuche im Rahmen einer Reevaluation wird durch Akronyme erleichtert.

$\square$ Reevaluation nach D O P E S und A B C D E

\section{Erhalte die Narkose aufrecht, beatme protektiv}

$\square$ AZV: $6-8 \mathrm{ml} / \mathrm{kg}$, Pinsp. und $\mathrm{FiO}_{2}$ titrieren, Ziel-SpO 2 : mind. 94\%

$\square$ etCO ${ }_{2}: 35 \mathrm{mmHg}$, Respiratorbeatmung* erst > $10 \mathrm{~kg} \mathrm{KG}$ sicher möglich

$\square$ kontinuierliche Aufrechterhaltung der Narkose mittels Perfusor

( ${ }^{*}$ aktuelle Notfallrespiratoren im Rettungsdienst)

\section{Temperaturkontrolle}

$\square$ Vermeide akzidentelle Hyothermie

23 Landsleitner B, Schroth M. Analgesie und Anästhesie bei Kindernotfällen. Notfall Rettungsmed 2014; 17: 95-104

24 Adams HA, Flemming A. Analgesie, Sedierung und Anästhesie in der Notfallmedizin. Anästh Intensivmed 2015; 56: 75-90

25 Böhm R, Meybohm P, Kunz T. Ketamin - bewährtes Narkotikum mit neuen Indikationen. Notfallmedizin up2date 2014: 9: 292-293

26 Tarquinio KM, Howell JD, Montgomery V et al. (2015): Current Medication Practice and Tracheal Intubation Safety Outcomes from a Prospective Multicenter Observational Cohort Study; Pediatr Crit Care Med 2015; 9: 210-218

27 Keil J, Jung P, Schiele A et al. Interdisziplinär konsentierte Stellungnahme zum Atemwegsmanagement mit supraglottischen Atemwegshilfen in der Kindernotfallmedizin. Anästhesist 2016; 65: 57-66

28 Kaufmann J, Laschat M, Wappler F. Medikamentenfehler bei Kindernotfällen: eine systematische Analyse. Dtsch Arztebl Int 2012;109: 609-616

Beitrag online zu finden unter http://dx.doi.org/10.1055/s-0042-117356 